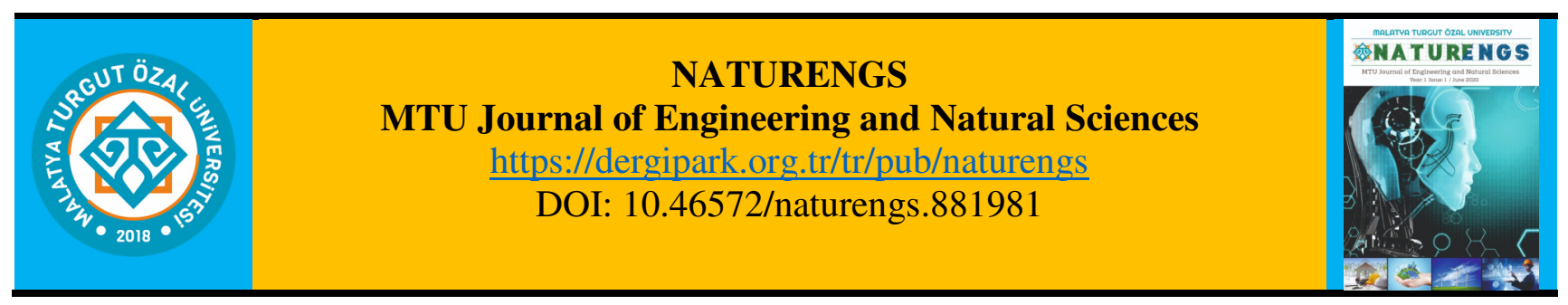

Research Article

\title{
Some Inequalities on Half Lightlike Submanifolds of a Lorentzian Manifold with Semi-Symmetric Metric Connection
}

\author{
Nergiz (ÖNEN) POYRAZ ${ }^{1}$, Burçin DOĞAN ${ }^{2 *}$ \\ ${ }^{1}$ Department of Mathematics, Faculty of Arts and Sciences, Çukurova University, Adana, Turkey. \\ 2* Department of Engineering Basic Science, Faculty of Engineering and Natural Sciences, Malatya Turgut Özal \\ University, Malatya, Turkey.
}

(Received: 17.02.2021; Accepted: 24.02.2021)

\begin{abstract}
In this paper, we introduce some inequalities for screen homothetic half lightlike submanifolds of a real space form $\tilde{M}(c)$ of constant sectional curvature $c$, endowed with the semi-symmetric metric connection. Using these inequalities, we derive some characterizations for such half lightlike submanifolds. Finally, ChenRicci inequalities are calculated. Moreover, the equality cases are considered and we get some results.
\end{abstract}

Keywords: Chen inequalities, Half lightlike submanifold, Lorentzian manifold, Semi-symmetric metric connection.

\section{INTRODUCTION}

Lightlike geometry has applications in mathematical physics and because of this, it is an important research field in differential geometry. Kupeli initiated the geometry of lightlike submanifolds in [1]. Then Duggal and Bejancu developed it [2]. Moreover, many authors studied it $[3,4]$.

A semi-symmetric linear connection on a differentiable manifold is presented in [5], the geometers studied it in [6] and [7]. Then, in [8] and [9], the geometry of a Riemannian manifold and a hypersurface of a Riemannian manifold with a semi-symmetric metric connection was investigated by Imai. The studies on Riemannian, semi-Riemannian, and Lorentzian manifolds with semi-symmetric metric connections belong to Nakao [10], Duggal-Sharma [11], and Konar-Biswas [12], respectively. Lightlike hypersurfaces of a semi-Riemannian manifold with a semi-symmetric metric connection were introduced in [13]. Later, Akyol, Vanlı, and Fernandez investigated the geometry of such connection on S manifolds in [14].

In addition to these, Chen introduced Chen inequalities and defined new types of curvature invariants in [15], and then, many authors worked on this topic [16-23]. Chen inequalities on submanifolds with constant and quasi-constant curvature with a semi-symmetric metric connection were studied in [24] and [25], respectively.

Firstly Chen inequalities on lightlike geometry were worked by Gülbahar, Kılıç, and Keleş in

${ }^{*}$ Corresponding Author: burcin.dogan@ozal.edu.tr

ORCID number of authors: ${ }^{1} 0000-0002-8110-712 \mathrm{X},{ }^{2}$ 0000-0001-8386-213X 
[26] and [27]. Then Poyraz, Doğan, and Yaşar studied Chen inequalities on the lightlike hypersurface of a Lorentzian manifold with a semi-symmetric metric connection in [28]. Some inequalities for screen conformal half lightlike submanifolds were established by Gülbahar and K1lıç in [29].

In this study, we introduce some inequalities for screen homothetic half lightlike submanifolds of a real space form $\tilde{M}(c)$ endowed with the semi-symmetric metric connection. Using these inequalities, we derive some characterizations for such half lightlike submanifolds. Finally, Chen-Ricci inequalities are calculated. Moreover, the equality cases are considered and we get some results.

\section{PRELIMINARIES}

Let $(\tilde{M}, \tilde{g})$ be a semi-Riemannian manifold. A connection $\tilde{\nabla} \tilde{M}$ is called a semi-symmetric metric connection if it is metric, i.e., $\tilde{\nabla} \tilde{g}=0$ and its torsion tensor $\tilde{T}$ satisfies

$\tilde{T}(\tilde{X}, \tilde{Y})=\tilde{\pi}(\tilde{Y}) \tilde{X}-\tilde{\pi}(\tilde{X}) \tilde{Y}$

for any vector fields $\tilde{X}$ and $\tilde{Y}$ of $\tilde{M}$, where $\tilde{\pi}$ is a 1-form defined by

$\tilde{g}(\tilde{P}, \tilde{X})=\tilde{\pi}(\tilde{X})$

and $\tilde{P}$ is a vector field on $\tilde{M}$, which is called the torsion vector field.

Let $\tilde{M}$ be a semi-Riemannian manifold admits a semi-symmetric metric connection which is given by

$\tilde{\nabla}_{\tilde{X}} \tilde{Y}=\tilde{\nabla}_{\tilde{X}} \tilde{Y}+\tilde{\pi}(\tilde{Y}) \tilde{X}-\tilde{g}(\tilde{X}, \tilde{Y}) \tilde{P}$

for any $\tilde{X}, \tilde{Y} \in \Gamma(T \tilde{M})$, where $\dot{\nabla}$ is the Levi-Civita connection concerning the semi-Riemannian metric $\tilde{g}$ [7].

Let $(\tilde{M}, \tilde{g})$ be a $(n+3)$-dimensional semi-Riemannian manifold of the index $q \geq 1$ and $M$ be a lightlike submanifold of codimension 2 of $\tilde{M}$. Then the radical distribution $\operatorname{Rad}(T M)=T M \cap T M^{\perp} \quad M$ is a vector subbundle of $T M$ and the $T M^{\perp}$ of rank 1 or 2 . If $\operatorname{rank}(\operatorname{Rad}(T M))=1$, then $M$ is called half lightlike submanifold of $\tilde{M}$. Then there exist complementary non-degenerate distributions $S(T M)$ and $S\left(T M^{\perp}\right)$ of $\operatorname{Rad}(T M)$ in $T M$ and $T M^{\perp}$, which are named the screen and the screen transversal distribution on $M$, respectively. Hence we derive

$T M=\operatorname{Rad}(T M) \perp S(T M), T M^{\perp}=\operatorname{Rad}(T M) \perp S\left(T M^{\perp}\right)$.

Consider the orthogonal complementary distribution $S(T M)^{\perp}$ to $S(T M)$ in $T \tilde{M}$. Then $\xi$ and $L$ belong to $\Gamma\left(S(T M)^{\perp}\right)$. Hence we obtain

$S(T M)^{\perp}=S\left(T M^{\perp}\right) \perp S\left(T M^{\perp}\right)^{\perp}$,

where $S\left(T M^{\perp}\right)^{\perp}$ is the orthogonal complementary to $S\left(T M^{\perp}\right)$ in $S(T M)^{\perp}$. For any null section $\xi \in \operatorname{Rad}(T M)$ on a coordinate neighborhood $U \subset M$, there exists a uniquely determined null vector field $N \in \Gamma(\operatorname{ltr}(T M))$ holding

$\tilde{g}(N, \xi)=1, \quad \tilde{g}(N, N)=\tilde{g}(N, X)=\tilde{g}(N, L)=0, \forall X \in \Gamma(T M)$. 
We say $\operatorname{ltr}(T M)$ and $\operatorname{tr}(T M)=S\left(T M^{\perp}\right) \perp \operatorname{ltr}(T M)$ the lightlike transversal vector bundle and transversal vector bundle $M$ for $S(T M)$, respectively. Hence we have

$$
\begin{aligned}
T \tilde{M} & =T M \oplus \operatorname{tr}(T M) \\
& =\{\operatorname{Rad}(T M) \oplus \operatorname{ltr}(T M)\} \perp S(T M) \perp S\left(T M^{\perp}\right) .
\end{aligned}
$$

Using (2.6) we define the projection morphism $Q: \Gamma(T M) \rightarrow \Gamma(S(T M))$. Hence we have

$\tilde{\nabla}_{X} Y=\nabla_{X} Y+B(X, Y) N+D(X, Y) L$

$\tilde{\nabla}_{X} U=-A_{U} X+\nabla_{X}^{t} U$

$\tilde{\nabla}_{X} N=-A_{N} X+\tau(X) N+\rho(X) L$

$\tilde{\nabla}_{X} L=-A_{L} X+\psi(X) N$,

$\nabla_{X} Q Y=\nabla_{X}^{*} Q Y+C(X, Q Y) \xi$

$\nabla_{X} \xi=-A_{\xi}^{*} X-\tau(X) \xi$,

for any $X, Y \in \Gamma(T M), \xi \in \Gamma(\operatorname{Rad}(T M)), U \in \Gamma(\operatorname{tr}(T M)), N \in \Gamma(\operatorname{ltr}(T M))$ and $L \in \Gamma\left(S\left(T M^{\perp}\right)\right)$.

Then $\nabla$ and $\nabla^{*}$ are named induced linear connections on $T M$ and $S(T M)$ respectively, $B$ and $D$ are named the local second fundamental forms of $M, C$ is named the local second fundamental form on $S(T M) . A_{N}, A_{\xi}^{*}$ and $A_{L}$ are named linear operators on $T M$. Also $\tau, \rho$ and $\psi$ are named $1-$ forms on $T M$.

This $\nabla$ is not a metric connection and holds

$\left(\nabla_{X} g\right)(Y, Z)=B(X, Y) \eta(Z)+B(X, Z) \eta(Y)$,

for any $X, Y, Z \in \Gamma(T M)$, where $\eta$ is a $1-$ form defined by

$\eta(X)=\tilde{g}(X, N), \forall X \in \Gamma(T M)$.

But $\nabla^{*}$ is a metric connection. Using (2.1) and (2.13), we see that

$T(X, Y)=\pi(Y) X-\pi(X) Y$

and $B$ and $D$ are symmetric, where $T$ is the torsion tensor for $\nabla$. From (2.13) and (2.15), we see that $\nabla$ is a semi-symmetric non-metric connection of $M$. Moreover, $B$ and $D$ are independent of the choice of $S(T M)$ and hold

$$
B(X, \xi)=0, D(X, \xi)=-\varepsilon \psi(X), \forall X \in \Gamma(T M) \text {. }
$$

Therefore one obtains

$$
\begin{aligned}
& B(X, Y)=g\left(A_{\xi}^{*} X, Y\right), \quad g\left(A_{\xi}^{*} X, N\right)=0, \\
& C(X, Q Y)=g\left(A_{N} X, Q Y\right), \quad g\left(A_{N} X, N\right)=0, \\
& D(X, Q Y)=g\left(A_{L} X, Q Y\right), \quad g\left(A_{L} X, N\right)=\rho(X), \\
& D(X, Y)=g\left(A_{L} X, Y\right)-\psi(X) \eta(Y), \quad \forall X, Y \in \Gamma(T M) .
\end{aligned}
$$

By (2.17) and (2.18), $A_{\xi}^{*}$ and $A_{N}$ are $\Gamma(S(T M))$ - valued shape operators related to $B$ and $E$, respectively and $A_{\xi}^{*} \xi=0$.

Using (2.7), (2.12), and (2.16), one derives

$\tilde{\nabla}_{X} \xi=-A_{\xi}^{*} X-\tau(X) \xi-\varepsilon \psi(X) L$

for any $X \in \Gamma(T M)$. 
Definition 1. A half lightlike submanifold $(M, g)$ of a semi-Riemannian manifold $(\tilde{M}, \tilde{g})$ is named irrotational [1] if $\tilde{\nabla}_{X} \xi \in \Gamma(T M)$ for any $X \in \Gamma(T M)$. From (2.16) and (2.21), the definition of irrotational is equivalent to the condition $\psi(X)=0$, that $i s, D(X, \xi)=0$ for any $X \in \Gamma(T M)$.

Definition 2. A half lightlike submanifold $(M, g)$ of a semi-Riemannian manifold $(\tilde{M}, \tilde{g})$ is called umbilical in $\tilde{M}$ if there is a smooth vector field $H \in \Gamma(\operatorname{tr}(T M))$ on any coordinate neighborhood $U$ such that

$h(X, Y)=H g(X, Y)$

for any $X, Y \in \Gamma(T M)$, where

$h(X, Y)=B(X, Y) N+D(X, Y) L$

is the global second fundamental form tensor of $M$. In the case of $h=0$ on $U$, we call that $M$ is totally geodesic [30].

Besides, $M$ is totally umbilical iff, on each coordinate neighborhood $U$, there exist smooth vector functions $\lambda$ and $\delta$ such that

$B(X, Y)=\lambda g(X, Y), D_{2}(X, Y)=\delta g(X, Y)$,

for any $X, Y \in \Gamma(T M)$.

Definition 3. [30] The screen distribution $S(T M)$ of $M$ is named totally umbilical if there is a smooth function $\gamma$ on any coordinate neighborhood $U \subset M$ such that

$E(X, Q Y)=\gamma g(X, Y)$,

for any $X, Y \in \Gamma(T M)$. If $\gamma=0$ on $U$, then we say that $S(T M)$ is totally geodesic in $M$.

Furthermore, $(M, g, S(T M))$ is named minimal if $\psi(X)=0$ and

trace $_{S(T M)} h=0$,

where $\operatorname{trace}_{S(T M)}$ denotes the trace restricted to $S(T M)$ concerning the degenerate metric $g$ [31].

Let $(M, g, S(T M))$ be a $(n+1)$ - dimensional half-lightlike submanifold and $\left\{e_{1}, \ldots, e_{n}\right\}$ be an orthonormal basis of $\Gamma(S(T M))$. Let us consider

$\mu_{1}=\frac{1}{n} \sum_{j=1}^{n} B\left(e_{j}, e_{j}\right), \mu_{2}=\frac{1}{n} \sum_{j=1}^{n} D\left(e_{j}, e_{j}\right)$.

Then it is clear from (2.26) and (2.27) that $M$ is minimal iff $\mu_{1}=\mu_{2}=0$.

A lightlike hypersurface $(M, g)$ of a semi-Riemannian manifold $(\tilde{M}, \tilde{g})$ is called screen locally conformal if the shape operators $A_{N}$ and $A_{\xi}^{*}$ of $M$ and $S(T M)$, respectively, are related by

$A_{N}=\phi A_{\xi}^{*}$,

i.e.,

$C(X, P Y)=\phi B(X, Y), \forall X, Y \in \Gamma(T M)$,

where $\phi$ is a non-vanishing smooth function on a neighborhood $U$ in $M$. If $\phi$ is a non-zero constant, $M$ is named screen homothetic [32].

We denote $\tilde{R}, R$ and $R^{*}$ the curvature tensors of the semi-symmetric metric connection of $\tilde{\nabla}$, $\nabla$ and $\nabla^{*}$, respectively. 
Using (2.7)-(2.12) for $M$ and $S(T M)$, we derive:

$$
\begin{aligned}
\tilde{g}(\tilde{R}(X, Y) Z, Q W)= & g(R(X, Y) Z, Q W) \\
& +B(X, Z) C(Y, Q W)-B(Y, Z) C(X, Q W) \\
& +D(X, Z) D(Y, Q W)-D(Y, Z) D(X, Q W), \\
\tilde{g}(\tilde{R}(X, Y) Z, \xi)=\left(\nabla_{X} B\right)(Y, Z)-\left(\nabla_{Y} B\right)(X, Z) & +[\tau(X)-\pi(X)] B(Y, Z)-[\tau(Y)-\pi(Y)] B(X, Z) \\
+ & \psi(X) D(Y, Z)-\psi(Y) D(X, Z), \\
\tilde{g}(\tilde{R}(X, Y) Z, N)= & g(R(X, Y) Z, N) \\
+ & \rho(Y) D(X, Z)-\rho(X) D(Y, Z), \\
\tilde{g}(\tilde{R}(X, Y) \xi, N)= & g\left(A_{\xi}^{*} X, A_{N} Y\right)-g\left(A_{\xi}^{*} Y, A_{N} X\right) \\
- & 2 d \tau(X, Y)+\rho(X) \psi(Y)-\rho(Y) \psi(X), \\
g(R(X, Y) Q Z, Q W)= & g\left(R^{*}(X, Y) Z, Q W\right)+B(Y, Q W) C(X, Q Z) \\
& -B(X, Q W) C(Y, Q Z), \\
\tilde{g}(R(X, Y) Q Z, N)= & \left(\nabla_{X} C\right)(Y, Q Z)-\left(\nabla_{Y} C\right)(X, Q Z) \\
& +[\tau(Y)+\pi(Y)] C(X, Q Z)-[\tau(X)+\pi(X)] C(Y, Q Z),
\end{aligned}
$$

for any $X, Y, Z \in \Gamma(T M)[33]$.

Now let us choose a 2-dimensional non-degenerate plane section

$\Pi=\operatorname{Span}\{X, Y\}$,

in $T_{p} M, p \in M$. Then the sectional curvature at $p$ is expressed by [34]

$$
K(\Pi)=\frac{g(R(X, Y) Y, X)}{g(X, X) g(Y, Y)-g(X, Y)^{2}} .
$$

Let $p \in M$ and $\xi$ be the null vector of $T_{p} M$. A plane $\Pi$ of $T_{p} M$ is said to be null plane if it contains $\xi$ and $e_{i}$ such that $g\left(\xi, e_{i}\right)=0$ and $g\left(e_{i}, e_{i}\right)=\varepsilon_{i}= \pm 1$. The null sectional curvature of $\Pi$ is defined by

$$
K_{i}^{\text {null }}=\frac{g\left(R_{p}\left(e_{i}, \xi\right) \xi, e_{i}\right)}{g_{p}\left(e_{i}, e_{i}\right)} .
$$

The Ricci tensor Ric of $\tilde{M}$ and the induced Ricci type tensor $R^{(0,2)}$ of $M$ are given by

$$
\begin{aligned}
& \operatorname{Ric}(X, Y)=\operatorname{trace}\{Z \rightarrow \tilde{R}(Z, X) Y\}, \forall X, Y \in \Gamma(T \tilde{M}), \\
& R^{(0,2)}(X, Y)=\operatorname{trace}\{Z \rightarrow R(Z, X) Y\}, \forall X, Y \in \Gamma(T M) \text {, }
\end{aligned}
$$

where

$$
R^{(0,2)}(X, Y)=\sum_{i=1}^{n} \varepsilon_{i} g\left(R\left(e_{i}, X\right) Y, e_{i}\right)+\tilde{g}(R(\xi, X) Y, N)
$$

for the quasi-orthonormal frame $\left\{e_{1}, \ldots, e_{n}, \xi\right\}$ of $T_{p} M$. From the equations (2.30)-(2.33), it can be shown that the Ricci type tensor doesn't need to be symmetric as the sectional curvature map. This tensor is called Ricci tensor if it is symmetric.

One defines scalar curvature $\tau$ by

$$
\tau(p)=\sum_{i, j=1}^{n} K_{i j}+\sum_{i=1}^{n} K_{i}^{\text {null }}+K_{i N}
$$


where $K_{i N}=\tilde{g}\left(R\left(\xi, e_{i}\right) e_{i}, N\right)$ for $i \in\{1, \ldots, n\}$.

\section{CHEN RICCI INEQUALITIES}

Let $M$ be a $(n+1)$-dimensional half lightlike submanifold of a $(n+3)$-dimensional of a Lorentzian manifold $\tilde{M}$ with a semi-symmetric metric connection and $\left\{e_{1}, \ldots ., e_{n}, \xi\right\}$ be a basis of $\Gamma(T M)$ where $\left\{e_{1}, \ldots ., e_{n}\right\}$ be an orthonormal basis of $\Gamma(S(T M))$. For $k \leq n$, we establish $\pi_{k, \xi}=s p\left\{e_{1}, \ldots ., e_{k}, \xi\right\}$ is a $(k+1)-$ dimensional degenerate plane section and $\pi_{k}=s p\left\{e_{1}, \ldots ., e_{k}\right\}$ is $k$-dimensional non-degenerate plane section. The $k$-degenerate Ricci curvature and the $k-$ Ricci curvature are defined by

$$
\begin{aligned}
& \operatorname{Ric}_{\pi_{k, \xi}}(X)=R^{(0,2)}(X, X)=\sum_{j=1}^{k} g\left(R\left(e_{j}, X\right) X, e_{j}\right)+\hat{g}(R(\xi, X) X, N), \\
& R i c_{\pi_{k}}(X)=R^{(0,2)}(X, X)=\sum_{j=1}^{k} g\left(R\left(e_{j}, X\right) X, e_{j}\right),
\end{aligned}
$$

respectively for a unit vector $X \in \Gamma(T M)$. Also, $k$-degenerate scalar curvature and $k$-scalar curvature at $p \in M$ are given by

$$
\begin{aligned}
& \tau_{\pi_{k, \xi}}(p)=\sum_{i, j=1}^{k} K_{i j}+\sum_{i=1}^{k} K_{i}^{\text {null }}+K_{i N}, \\
& \tau_{\pi_{k}}(p)=\sum_{i, j=1}^{k} K_{i j},
\end{aligned}
$$

respectively. For $k=n, \pi_{n}=\operatorname{sp}\left\{e_{1}, \ldots, e_{n}\right\}=\Gamma(S(T M))$, we have the screen Ricci curvature and the screen scalar curvature given by

$$
\operatorname{Ric}_{S(T M)}\left(e_{1}\right)=\operatorname{Ric}_{\pi_{n}}\left(e_{1}\right)=\sum_{j=1}^{n} K_{1 j}=K_{12}+\ldots+K_{1 n},
$$

and

$$
\tau_{S(T M)}=\sum_{i, j=1}^{n} K_{i j}
$$

respectively.

Let $\tilde{M}(c)$ be a real space form of constant sectional curvature $c$ endowed with a semisymmetric metric connection $\tilde{\nabla}$. The curvature tensor $\stackrel{\circ}{\tilde{R}}$ to the Levi-Civita connection $\stackrel{\circ}{\tilde{\nabla}}$ on $\tilde{M}(c)$ is defined by

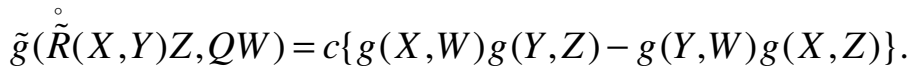

Using (2.2), we derive

$$
\begin{aligned}
\tilde{g}(\tilde{R}(X, Y) Z, Q W)= & \tilde{g}(\tilde{\tilde{R}}(X, Y) Z, Q W)-\alpha(Y, Z) g(X, W)+\alpha(X, Z) g(Y, W) \\
& -\alpha(X, W) g(Y, Z)+\alpha(Y, W) g(X, Z),
\end{aligned}
$$

for any $X, Y, Z, W \in \Gamma(T M)$, where $\alpha$ is a $(0,2)$ tensor field defined by

$\alpha(X, Y)=\left(\tilde{\nabla}_{X} \pi\right) Y-\pi(X) \pi(Y)+\frac{1}{2} \pi(Q) g(X, Y)$

$[8]$. 
From (2.30), (3.6), (3.7), and (3.8), we can write

$\tau_{S(T M)}(p)=n(n-1) c-2(n-1) \lambda+\sum_{i, j=1}^{n} B_{i i} C_{j j}-B_{i j} C_{j i}+\sum_{i, j=1}^{n} D_{i i} D_{j j}-D_{i j} D_{j i}$,

where $\lambda$ is the trace of $\alpha$ and $B_{i j}=B\left(e_{i}, e_{j}\right), C_{i j}=C\left(e_{i}, e_{j}\right), D_{i j}=D\left(e_{i}, e_{j}\right)$ for $i, j \in\{1, \ldots, n\}$.

Let $M$ be a screen homothetic half lightlike submanifold of a $(n+3)$-dimensional Lorentzian space form $\tilde{M}(c)$. Using (2.29) and (3.10) we get

$$
\tau_{S(T M)}(p)=n(n-1) c-2(n-1) \lambda+\phi n^{2} \mu_{1}^{2}+n^{2} \mu_{2}^{2}-\sum_{i, j=1}^{n}\left[\phi\left(B_{i j}\right)^{2}+\left(D_{i j}\right)^{2}\right] .
$$

Lemma 4. [35] Let $a_{1}, \ldots, a_{n}$ be $n$ - real numbers and define $A=\sum_{i<j}\left(a_{i}-a_{j}\right)^{2}$. Then

(1) $A \geq \frac{n}{2}\left(a_{1}-a_{2}\right)^{2}$ and equality holds iff

$\frac{1}{2}\left(a_{1}+a_{2}\right)=a_{3}=\ldots=a_{n}$.

(2) Let $k, \ell$ be integers such that $1 \leq k<\ell \leq n$ and $(k, \ell) \neq(1,2)$. If $A=\frac{n}{2}\left(a_{1}-a_{2}\right)^{2}=\frac{n}{2}\left(a_{k}-a_{1}\right)^{2}$ then $a_{1}=a_{2}=\ldots=a_{n}$.

Theorem 5. Let $M$ be a $(n+1)$-dimensional screen homothetic half lightlike submanifold with $\phi>0$ of a $(n+3)$-dimensional Lorentzian space form $\tilde{M}(c)$ endowed with a semi-symmetric metric connection $\tilde{\nabla}$. Then we obtain

$$
\begin{aligned}
\tau_{S(T M)}(p) \leq & n(n-1) c-2(n-1) \lambda+\frac{n^{3}}{n+1} \phi \mu_{1}^{2} \\
& -\frac{\phi n}{2(n+1)} \sum_{i, j=1}^{n}\left(B_{11}-B_{22}\right)^{2}+\frac{n^{3}}{n+1} \mu_{2}^{2} \\
& -\frac{n}{2(n+1)} \sum_{i, j=1}^{n}\left(D_{11}-D_{22}\right)^{2} .
\end{aligned}
$$

The equality case of (3.12) satisfies at $p \in M$ iff

$\mu_{1}=\frac{n}{2}\left(B_{11}+B_{22}\right)^{2}, \mu_{2}=\frac{n}{2}\left(D_{11}+D_{22}\right)^{2}$

and

$$
B_{i j}=D_{i j}=0 \text {, for } i \neq j \in\{1, \ldots, n\} \text {. }
$$

Proof. From the Binomial theorem, we can write

$$
\begin{gathered}
\left(B_{11}-B_{22}\right)^{2}+\ldots+\left(B_{11}-B_{n n}\right)^{2}+\left(B_{22}-B_{33}\right)^{2}+\ldots+\left(B_{22}-B_{n n}\right)^{2} \\
+\ldots+\left(B_{n-1 n-1}-B_{n n}\right)^{2}=n \sum_{i=1}^{n}\left(B_{i i}\right)^{2}-2 \sum_{i \neq j}^{n} B_{i i} B_{j j} .
\end{gathered}
$$

By Lemma 4 and (3.15) we obtain

$$
\sum_{i=1}^{n}\left(B_{i i}\right)^{2} \geq \frac{1}{n} \sum_{i \neq j} B_{i i} B_{j j}+\frac{1}{2}\left(B_{11}-B_{22}\right)^{2} \text {. }
$$

We also can derive

$$
\frac{2}{n} \sum_{i \neq j} B_{i i} B_{j j}=n \mu_{1}^{2}-\frac{1}{n} \sum_{i=1}^{n}\left(B_{i i}\right)^{2} \text {. }
$$

Using (3.16) and (3.17) we get 


$$
\sum_{i=1}^{n}\left(B_{i i}\right)^{2} \geq \frac{n^{2}}{n+1} \mu_{1}^{2}+\frac{n}{2(n+1)}\left(B_{11}-B_{22}\right)^{2}
$$

Similarly, we obtain

$$
\sum_{i=1}^{n}\left(D_{i i}\right)^{2} \geq \frac{n^{2}}{n+1} \mu_{2}^{2}+\frac{n}{2(n+1)}\left(D_{11}-D_{22}\right)^{2} .
$$

Finally, using (3.18) and (3.19) in (3.11), we obtain (3.12).

Taking consideration of case (1) of Lemma 4, the equality of (3.12) holds at $p \in M$ iff (3.13) and (3.14) hold. The converse part of the theorem is straightforward.

Considering Theorem 5, we derive Corollary 6 and Corollary 7.

Corollary 6. Let $M$ be a $(n+1)$-dimensional screen homothetic half lightlike submanifold with $\phi>0$ of a $(n+3)$-dimensional Lorentzian space form $\tilde{M}(c)$ endowed with a semisymmetric metric connection $\tilde{\nabla}$. Then we have

$$
\begin{aligned}
\tau_{S(T M)}(p) \leq & n(n-1) c-2(n-1) \lambda+\frac{n^{3}}{n+1} \phi \mu_{1}^{2}-\frac{\phi n}{2(n+1)} \sum_{i, j=1}^{n}\left(B_{i i}-B_{j j}\right)^{2} \\
& +\frac{n^{3}}{n+1} \mu_{2}^{2}-\frac{n}{2(n+1)} \sum_{i, j=1}^{n}\left(D_{i i}-D_{j j}\right)^{2} .
\end{aligned}
$$

The equality case of (3.20) satisfies for all $p \in M$ iff $S(T M)$ is a totally umbilical in $M$.

Corollary 7. Let $M$ be a $(n+1)$-dimensional irrotational screen homothetic half lightlike submanifold with $\phi>0$ of a $(n+3)$-dimensional Lorentzian space form $\tilde{M}(c)$ endowed with a semi-symmetric metric connection $\tilde{\nabla}$. Then we have

$$
\begin{aligned}
\tau_{S(T M)}(p) \leq & n(n-1) c-2(n-1) \lambda+\frac{n^{3}}{n+1} \phi \mu_{1}^{2} \\
& -\frac{\phi n}{2(n+1)} \sum_{i, j=1}^{n}\left(B_{i i}-B_{j j}\right)^{2}+\frac{n^{3}}{n+1} \mu_{2}^{2}-\frac{n}{2(n+1)} \sum_{i, j=1}^{n}\left(D_{i i}-D_{j j}\right)^{2} .
\end{aligned}
$$

The equality case of (3.21) holds for all $p \in M$ iff $M$ is totally umbilical.

Lemma 8. [15] If $n \geq 2$ and $a_{1}, \ldots, a_{n} \in \square$ are real numbers such that

$$
\left(\sum_{i=1}^{n} a_{i}\right)^{2}=(n-1)\left(\sum_{i=1}^{n} a_{i}^{2}+a\right) \text {, }
$$

then

$2 a_{1} a_{2} \geq a$

with equality holding iff

$a_{1}+a_{2}=a_{3}=\ldots=a_{n}$.

Theorem 9. Let $M$ be a $(n+1)$-dimensional screen homothetic half lightlike submanifold with $\phi>0$ of a $(n+3)$-dimensional Lorentzian space form $\tilde{M}(c)$ endowed with a semi-symmetric metric connection $\tilde{\nabla}$ such that the vector field $P$ is tangent to $M$ and $\Pi=\operatorname{Span}\left\{e_{1}, e_{2}\right\}$ be a 2-dimensional non-degenerate plane section of $T_{p} M, p \in M$. Then we have 


$$
\begin{aligned}
\tau_{S(T M)}(p)-\tau(\Pi) \leq & (n-2)\left(\frac{\phi n^{2}}{n-1} \mu_{1}^{2}+(n+1) c-2 \lambda\right) \\
& -2 \operatorname{trace}\left(\left.\lambda\right|_{\Pi^{\perp}}\right)+B\left(\Pi^{\perp}\right)^{2}+{ }^{2} n^{2} \mu_{2}^{2}\left(\Pi^{\perp}\right) \\
& +\sum_{j>2}^{n} D_{11} D_{j j}+D_{22} D_{j j}
\end{aligned}
$$

where

$$
B\left(\Pi^{\perp}\right)^{2}=\sum_{i=3}^{n}\left(B_{i i}\right)^{2} \text { and } n^{2} \mu_{2}^{2}\left(\Pi^{\perp}\right)=\sum_{i, j=3}^{n} D_{i i} D_{j j} .
$$

The equality case of (3.22) holds for all $p \in M$ if $\mu_{1}=0, \mu_{2}=D_{11}+D_{22}$ and the shape operator $A_{\xi}^{*}$ of $M$

$$
A_{\xi}^{*}=\left(\begin{array}{cccc}
B_{11} & B_{12} & \ldots & 0 \\
B_{21} & B_{22} & \ldots & 0 \\
& & & \\
0 & 0 & \ldots & 0
\end{array}\right) .
$$

Proof. If

$$
\begin{aligned}
\delta= & \tau_{S(T M)}(p)-n(n-1) c+2(n-1) \lambda-\phi n^{2} \frac{(n-2)}{n-1} \mu_{1}^{2} \\
& -n^{2} \mu_{2}^{2}+\sum_{i, j=1}^{n}\left(D_{i j}\right)^{2}
\end{aligned}
$$

is said, we derive

$$
\delta=\phi \frac{n^{2}}{n-1} \mu_{1}^{2}-\phi \sum_{i, j=1}^{n}\left(B_{i j}\right)^{2} .
$$

Thus, we can derive

$$
\left(\sum_{i=1}^{n} B_{i i}\right)^{2}=(n-1)\left(\frac{\delta}{\phi}+\sum_{i=1}^{n}\left(B_{i i}\right)^{2}+\sum_{i \neq j=1}^{n}\left(B_{i j}\right)^{2}\right) \text {. }
$$

From Lemma 8, we obtain

$$
2 B_{11} B_{22} \geq \frac{\delta}{\phi}+\sum_{i \neq j=1}^{n}\left(B_{i j}\right)^{2}
$$

Let choose $\Pi=\operatorname{Sp}\left\{e_{1}, e_{2}\right\}$. Then, we get

$$
\begin{aligned}
\tau(\Pi)= & 2 c-2\left(\alpha_{11}+\alpha_{22}\right)+\phi \sum_{i, j=1}^{2} B_{i i} B_{j j}-\left(B_{i j}\right)^{2}+\sum_{i, j=1}^{2} D_{i i} D_{j j}-\left(D_{i j}\right)^{2} \\
\geq & 2 c-2\left(\alpha_{11}+\alpha_{22}\right)+\delta+\phi \sum_{i \neq j=1}^{n}\left(B_{i j}\right)^{2}-\phi \sum_{i \neq j=1}^{2}\left(B_{i j}\right)^{2} \\
& +\sum_{i, j=1}^{2} D_{i i} D_{j j}-\left(D_{i j}{ }^{2}\right) \\
= & 2 c-2\left(\alpha_{11}+\alpha_{22}\right)+\delta+\phi \sum_{i, j=1}^{n}\left(B_{i j}\right)^{2}-\phi \sum_{i=1}^{n}\left(B_{i i}\right)^{2} \\
& -\phi \sum_{i, j=1}^{2}\left(B_{i j}\right)^{2}-\phi \sum_{i=1}^{2}\left(B_{i i}\right)^{2}+\sum_{i, j=1}^{2} D_{i i} D_{j j}-\left(D_{i j}{ }^{2}\right) \\
\geq & 2 c-2\left(\alpha_{11}+\alpha_{22}\right)+\delta-\phi \sum_{i=3}^{n}\left(B_{i i}\right)^{2}+2 D_{11} D_{22}-\sum_{i \neq j}^{2}\left(D_{i j}\right)^{2}
\end{aligned}
$$

We remark that 
$\alpha\left(e_{1}, e_{1}\right)+\alpha\left(e_{2}, e_{2}\right)=\lambda-\operatorname{trace}\left(\left.\lambda\right|_{\Pi^{\perp}}\right)$.

Using (3.26), (3.29), and (3.30) we derive

$$
\begin{aligned}
\tau(\Pi) \geq & -(n-2)(n+1) c+2(n-1) \lambda-2\left(\lambda-\operatorname{trace}\left(\left.\lambda\right|_{\Pi^{\perp}}\right)\right)+\tau_{S(T M)}(p) \\
& -\phi n^{2} \frac{(n-2)}{(n-1)} \mu_{1}^{2}-\sum_{i, j=3}^{n} D_{i i} D_{j j}-\sum_{j>2}^{n}\left(D_{11} D_{j j}+D_{22} D_{j j}\right) \\
& +\sum_{i, j=3}^{n}\left(D_{i j}\right)^{2}-\phi \sum_{i=3}^{n}\left(B_{i i}\right)^{2} .
\end{aligned}
$$

Thus we obtain (3.22).

The equality case of (3.22) holds for all $p \in M$ iff for all $i, j \in\{3, \ldots, n\}$

$B_{i j}=D_{i j}=0$,

$B_{11}+B_{22}=B_{33}=0$.

Thus the proof is completed.

For the sectional curvature of screen conformal half lightlike submanifold is symmetric, the screen scalar curvature $r_{S(T M)}$ can be denoted by

$r_{S(T M)}(p)=\sum_{1 \leq i<j \leq n} K_{i j}=\frac{1}{2} \sum_{i, j=1}^{n} K_{i j}=\frac{1}{2} \tau_{S(T M)}(p)$.

By using (3.32), the equality (3.11) can be rewritten as follows:

$$
\begin{aligned}
2 r_{S(T M)}(p)= & n(n-1) c-2(n-1) \lambda+\phi n^{2} \mu_{1}^{2}+n^{2} \mu_{2}^{2} \\
& -\sum_{i, j=1}^{n}\left[\phi\left(B_{i j}\right)^{2}+\left(D_{i j}\right)^{2}\right] .
\end{aligned}
$$

Lemma 10. [36] Let $a_{1}, a_{2}, \ldots ., a_{n}$, be $n$-real number $(n>1)$, then

$\frac{1}{n}\left(\sum_{i=1}^{n} a_{i}\right)^{2} \leq \sum_{i=1}^{n} a_{i}^{2}$

with equality if $a_{1}=a_{2}=\ldots .=a_{n}$.

Theorem 11. Let $M$ be a $(n+1)$-dimensional screen conformal half lightlike submanifold with $\phi>0$ of a $(n+3)$-dimensional Lorentzian space form $\tilde{M}(c)$ endowed with a semisymmetric metric connection $\tilde{\nabla}$. Then we get

$2 r_{S(T M)}(p) \leq n(n-1)\left(c+\phi \mu_{1}^{2}+\mu_{2}^{2}\right)-2(n-1) \lambda$.

The equality of (3.34) satisfies for all $p \in M$ iff $S(T M)$ is a totally umbilical in $M$.

Proof. From (3.33), we have

$$
\begin{aligned}
2 r_{S(T M)}(p)= & n(n-1) c-2(n-1) \lambda+\phi n^{2} \mu_{1}^{2}-\phi \sum_{i=1}^{n}\left(B_{i i}\right)^{2} \\
& -\phi \sum_{i \neq j=1}^{n}\left(B_{i j}\right)^{2}+n^{2} \mu_{2}^{2}+\sum_{i=1}^{n}\left(D_{i i}\right)^{2}+\sum_{i \neq j=1}^{n}\left(D_{i j}\right)^{2} .
\end{aligned}
$$

Using Lemma 10 in (3.35) we find (3.34).

The equality of (3.34) holds for all $p \in M$ iff

$B_{11}=\ldots=B_{n n}, B_{i j}=0$,

$D_{11}=\ldots=D_{n n}, D_{i j}=0$, for $i \neq j \in\{1, \ldots, n\}$.

Thus $S(T M)$ is a totally umbilical in $M$. 
The following corollary is obtained from the previous theorem.

Corollary 12. Let $M$ be a $(n+1)$-dimensional irrational screen conformal half lightlike submanifold with $\phi>0$ of a $(n+3)$-dimensional Lorentzian space form $\tilde{M}(c)$ endowed with a semi-symmetric metric connection $\tilde{\nabla}$. Then we have

$2 r_{S(T M)}(p) \leq n(n-1)\left(c+\phi \mu_{1}^{2}+\mu_{2}^{2}\right)-2(n-1) \lambda$.

The equality of (3.36) holds for all $p \in M$ iff $M$ is totally umbilical.

One obtains the following equation from the Binomial theorem:

$$
\begin{aligned}
\sum_{i, j=1}^{n}\left(B_{i j}\right)^{2}= & \frac{1}{2} n^{2} \mu_{1}^{2}+\frac{1}{2}\left(B_{11}-B_{22}-\ldots-B_{n n}\right)^{2} \\
& +\sum_{j=2}^{n}\left(B_{1 j}\right)^{2}-\sum_{2 \leq i<j \leq n}^{m}\left(B_{i i} B_{j j}-\left(B_{i j}\right)^{2}\right) .
\end{aligned}
$$

Theorem 13. Let $M$ be a $(n+1)$-dimensional screen homothetic half lightlike submanifold with $\phi>0$ of a $(n+3)$-dimensional Lorentzian space form $\tilde{M}(c)$ endowed with a semisymmetric metric connection $\tilde{\nabla}$ such that the vector field $P$ is tangent to $M$. Then, the followings are true.

(i) For $X \in S^{1}(T M)=\{X \in S(T M):\langle X, X\rangle=1\}$

$\operatorname{Ric}_{S(T M)}(X) \leq \frac{1}{4}\left(\phi n^{2} \mu_{1}^{2}+n^{2} \mu_{2}^{2}\right)+(n-1) c-\lambda+(n-2) \alpha(X, X)$.

(ii) The equality case of (3.38) is held by $X \in S^{1}(T M)$ iff

$B(X, Y)=D(X, Y)=0$, for all $Y \in T_{p}(M)$ orthogonal to $X$,

$B(X, X)=\frac{n}{2} \mu_{1}, D(X, X)=\frac{n}{2} \mu_{2}$

(iii) The equality case of (3.38) holds for all $X \in S^{1}(T M)$ if either $S(T M)$ is totally geodesic in $M$ or $n=2$ and $S(T M)$ is totally umbilical in $M$.

Proof. From (3.33) and (3.37), we get

$$
\begin{aligned}
\frac{1}{4}\left(\phi n^{2} \mu_{1}^{2}+n^{2} \mu_{2}^{2}\right)= & r_{S(T M)}(p)-\frac{n(n-1)}{2} c+(n-1) \lambda \\
& +\frac{1}{4} \phi\left(B_{11}-B_{22}-\ldots-B_{n n}\right)^{2}+\phi \sum_{j=2}^{n}\left(B_{1 j}\right)^{2} \\
& -\phi \sum_{2 \leq i<j \leq n} B_{i i} B_{j j}-\left(B_{i j}\right)^{2}+\frac{1}{4}\left(D_{11}-D_{22}-\ldots-D_{n n}\right)^{2} \\
& +\sum_{j=2}^{n}\left(D_{1 j}\right)^{2}-\sum_{2 \leq i<j \leq n} D_{i i} D_{j j}-\left(D_{i j}\right)^{2} .
\end{aligned}
$$

Moreover,

$$
\begin{aligned}
& \phi \sum_{2 \leq i<j \leq n} B_{i i} B_{j j}-\left(B_{i j}\right)^{2}+\sum_{2 \leq i<j \leq n} D_{i i} D_{j j}-\left(D_{i j}\right)^{2} \\
= & \sum_{2 \leq i<j \leq n} K_{i j}-\frac{(n-2)(n-1)}{2} c+(n-2)\left(\lambda-\alpha\left(e_{1}, e_{1}\right)\right)
\end{aligned}
$$

is obtained. Using the two last equations, we have 


$$
\begin{aligned}
\operatorname{Ric}_{S(T M)}\left(e_{1}\right)= & \frac{1}{4}\left(\phi n^{2} \mu_{1}^{2}+n^{2} \mu_{2}^{2}\right)+(n-1) c-\lambda-(n-2) \alpha\left(e_{1}, e_{1}\right) \\
& -\frac{1}{4} \phi\left(B_{11}-B_{22}-\ldots-B_{n n}\right)^{2}-\phi \sum_{j=2}^{n}\left(B_{1 j}\right)^{2} \\
& -\frac{1}{4}\left(D_{11}-D_{22}-\ldots-D_{n n}\right)^{2}-\sum_{j=2}^{n}\left(D_{1 j}\right)^{2} .
\end{aligned}
$$

Choosing $e_{1}=X$ as any vector of $T_{p}^{1}(M)$ in (3.42) we get (3.38).

Equality holds in (3.38) for $X \in T_{p}^{1}(M)$ iff

$B_{12}=B_{13}=\ldots=B_{1 n}=0$ and $B_{11}=B_{22}+\ldots+B_{n n}$.

From (3.43) we obtain

$$
n \mu_{1}=B_{11}+\ldots+B_{n n}=2 B_{11} \text {. }
$$

Similarly, we get

$n \mu_{2}=D_{11}+\ldots+D_{n n}=2 D_{11}$.

From (3.44) and (3.45), we obtain (3.39).

Supposing the equality case of (3.38) for all $X \in T_{p}^{1}(M)$, considering (3.43), we derive $B_{i j}=0, \quad i \neq j$.

$2 B_{i i}=B_{11}+B_{22}+\ldots+B_{n n}, \quad i \in\{1, \ldots, n\}$.

From (3.47), we have $2 B_{11}=2 B_{22}=\ldots=2 B_{n n}=B_{11}+B_{22}+\ldots+B_{n n}$, which implies that $(n-2) \sum_{i=1}^{n} B_{i i}=0$.

Similarly, we get

$(n-2) \sum_{i=1}^{n} D_{i i}=0$.

Thus, either $\sum_{i=1}^{n} B_{i i}=\sum_{i=1}^{n} D_{i i}=0$ or $n=2$. If $\sum_{i=1}^{n} B_{i i}=\sum_{i=1}^{n} D_{i i}=0$, then from (3.47) we get $B_{i i}=D_{i i}=0, \forall i \in\{1, \ldots, n\}$.

From (3.46) and (3.48), we derive $B_{i j}=D_{i j}=0, \forall i, j \in\{1, \ldots, n\}$. Hence, $S(T M)$ is totally geodesic in $M$. If $n=2$, then from (3.47),

$2 B_{11}=2 B_{22}=B_{11}+B_{22}$,

$2 D_{11}=2 D_{22}=D_{11}+D_{22}$,

that is, $S(T M)$ is totally umbilical in $M$. The converse of proof is trivial.

\section{REFERENCES}

[1] Kupeli, D. N., (1996). Singular semi-Riemannian Geometry, Kluwer Academic Publishers, Dordrecht.

[2] Duggal, K. L., Bejancu, A., (1996). Lightlike Submanifolds of Semi-Riemannian Manifolds and Applications, Kluwer Academic Publishers, Dordrecht.

[3] Duggal, K. L., Jin, D. H. (2007). Null curves and Hypersurfaces of Semi-Riemannian Manifolds, World Scientific.

[4] Duggal, K. L. and Sahin, B., (2010). Differential Geometry of Lightlike Submanifolds, Birkhäuser, Basel.

[5] Friedmann, A and Schouten, J. A., (1924). Über die Geometrie der halbsymmetrischen Übertragungen, (German) Math. Z., 1(21): 211-223. 
[6] Hayden, H. A., (1932). Subspace of a space with torsion, Proceedings of the London Mathematical Society II Series, 34, 27-50.

[7] Yano, K., (1970). On Semi-Symmetric Metric Connection, Rev. Roum. Math. Pures Et Appl., 15: 1579-1586.

[8] Imai, T., (1972). Hypersurfaces of a Riemannian Manifold with Semi-Symmetric Metric Connection, Tensor, N.S., 23: 300-306.

[9] Imai, T., (1972). Notes on Semi-Symmetric Metric Connection, Tensor, N.S., 24: 293-296.

[10] Nakao, Z., (1976). Submanifolds of a Riemannian manifold with semi-symmetric metric connections, Proc. Amer. Math. Soc., 54: 261-266.

[11] Duggal, K. L., and Sharma, R., (1986). Semi-Symmetric metric connection in a Semi-Riemannian Manifold, Indian J. Pure appl Math., 17: 1276-1283.

[12] Konar, A. and Biswas, B., (2001). Lorentzian Manifold that Admits a type of Semi-Symmetric Metric Connection, Bull. Cal. Math., Soc., 93(5): 427-437.

[13] Yaşar, E., Çöken, A. C., Yücesan, A., (2007). Lightlike Hypersurfaces of Semi-Riemannian Manifold with Semi-Symmetric Metric Connection, Kuwait Journal of Science and Engineering, 34(2A): 11-24.

[14] Akyol, M. A., Vanl1, A. T. And Fernandez, L. M., (2013). Curvature properties of a semi symmetric metric connection on S manifolds., Annales Polonici Mathematici, 107(1): 71-86.

[15] Chen, B. Y., (1993). Some pinching and classification theorems for minimal submanifolds, Arch. math., (Basel), 60(6): 568-578.

[16] Chen, B. Y., (1998). Strings of Riemannian invariants, inequalities, ideal immersions and their applications, The Third Pacific Rim Geometry Conference (Seoul, 1996), 7-60, Monogr. Geom. Topology, 25, Int. Press, Cambridge, MA.

[17] Chen, B. Y., (2000). Some new obstructions to minimal and Lagrangian isometric immersions, Japanese J. Math., 26: 105-127.

[18] Chen, B. Y., (2008). $\delta$-invariants, Inequalities of Submanifolds and Their Applications, in Topics in Differential Geometry, Eds. A. Mihai, I. Mihai, R. Miron, Editura Academiei Romane, Bucuresti, 29-156.

[19] Chen, B. Y., Dillen, F., Verstraelen, L. and Vrancken, V., (2000). Characterizations of Riemannian space forms, Einstein spaces and conformally flat spaces, Proc. Amer. Math. Soc., 128: 589-598.

[20] Hong, S., Matsumoto K. and Tripathi, M. M., (2005). Certain basic inequalities for submanifolds of locally conformal Kaehlerian space forms, SUT J. Math., 4(1): 75-94.

[21] Kim, J. S., Choi, J., (2003). A basic inequality for submanifolds in a cosymplectic space form, Int. J. Math. Math. Sci., 9: 539-547.

[22] Matsumoto, K., Mihai, I., Oiaga, A., (2001). Ricci curvature of submanifolds in complex space forms, Rev. Roumaine Math. Pures Appl., 46: 775-782.

[23] Oiaga, A., Mihai, I., Chen, B. Y., (1999). Inequalities for slant submanifolds in complex space forms, Demonstratio Math., 32: 835-846.

[24] Mihai, A. and Özgür, C., (2010). Chen inequalities for submanifolds of real space form with a semi-symmetric metric connection, Taiwanese Journal of Mathematics, 14(4): 1465-1477.

[25] Zhang, P., Zhang, L. and Song, W., (2014). Chen's inequalities for submanifolds of a Riemannian manifold of quasi-constant curvature with a semi-symmetric metric connection, Taiwanese Journal of Mathematics, 18(6): 1841-1862. 
[26] Gülbahar, M., Kılıç, E. and Keleş, S., (2013). Chen-like inequalities on lightlike hypersurfaces of a Lorentzian manifold, J. Inequal. Appl., 266.

[27] Gülbahar, M., Kılıç, E. and Keleş, S., (2013). Some inequalities on screen homothetic lightlike hypersurfaces of a Lorentzian manifold, Taiwanese Journal of Mathematics, 17(6): 2083-2100.

[28] Poyraz, N. Ö., Doğan, B. and Yaşar, E., (2017). Chen Inequalities on Lightlike Hypersurface of a Lorentzian manifold with semi-symmetric metric connection, Int. Electronic Journal of Geometry, 10(1): 1-14.

[29] Gülbahar, M., Kılıç, E., (2017). Some optimal inequalities for screen conformal half-lightlike submanifolds, Acta Mathematica Academiae Paedagogicae Nyíregyháziensis, 33(2): 315-329.

[30] Duggal, K. L. and Jin, D. H., (1999). Half lightlike submanifolds of codimension 2, Math. J. Toyama Univ, 22: $121-161$

[31] Bejan, C. L. and Duggal, K. L., (2005). Global lightlike manifolds and harmonicity, Kodai Math. J., 28(1): 131-145.

[32] Duggal, K. L. and Sahin, B., (2004). Screen conformal half-lightlike submanifolds, Int. J. Math. and Math. Sci., 68: 3737-3753.

[33] Jin, D. H., (2011). Geometry of half lightlike submanifolds of a semi-Riemannian space form with a semisymmetric metric connection, J. Chungcheong Math. Soc., 24(4): 769-780.

[34] Beem, J. K., Ehrlich, P. E. and Easley, K. L., (1996). Global Lorentzian Geometry, Volume 202 of Monographs and Textbooks in Pure and Applied Mathematics, Marcel Dekker, Inc., New York.

[35] De Smet, P. J., Dillen, F., Verstraelen, L. and Vrancken, V., (1999). A pointwise inequality in submanifold theory, Arch. Math. (Brno), 5(2): 115-128.

[36] Tripathi, M. M., (2003). Certain Basic Inequalities for Submanifolds in ( ; ) Space, Recent Advances in Riemannian and Lorentzian Geometries, Baltimore, 187-202. 